MICROVAWE IMAGING OF MULTILAYER CYLINDERS USING OPTIMIZATION TECHNIQUES

\author{
Miguel Ferrando *, Antoni Broquetas, Luis Jof re, Juan M. Rius \\ E.T.S.I. Telecomunicacion, Apdo 30.002 \\ 08080-Barcelona, Spain
}

Introduction

The reconstruction of weak-scattering objects from the scattered field measured in cylindrical geometries, can be solved by using the Born aproximation [1]. However, first order algorithms of ten fail to reconstruct biological bodies due to their high contrast in permitivity [2].

The reconstruction of arbitrary strong-scattering objects is still an open area of research. The paper presents a new aproach to solve this problem using optimization techniques. The algorithm is based on the minimization of the mean squared error between the measured and calculated scattered fields from the reconstruction.

In this work the object is assumed to have cylindrical symmetry and we are interested in the radial profile of permittivity. The validity of the proposed algorithm is investigated both numerically and experimentally

Direct method: difraction of circular concentric cylinders

The theoretical scattering solution for a wave incident normally on a lossy dielectric multilayer circular cylinder can be obtained using the Richmond's recursive method [3].

For a TM mode, the E-field outside the cylinder is the sum of the incident and scattered.

$$
E_{z}(r, \phi)=\sum_{n=-\infty}^{n=\infty}\left(a_{n} J_{n}\left(k_{0} r\right)+c_{n} H_{n}^{(2)}\left(k_{0} r\right)\right) e^{j n \phi}
$$

In the layer $m$ the field is

$$
E_{z, m}(r, \phi)=\sum_{n=-\infty}^{n=\infty}\left(A_{m n} J_{n}\left(k_{m} r\right)+B_{m n} Y_{n}\left(k_{m} r\right)\right) e^{j n \varphi}
$$

Matching the tangential fields across the boundary, the recursion equation is obtained

$$
\begin{gathered}
\left(\begin{array}{l}
A_{m+1, n} \\
B_{m+1, n}
\end{array}\right)=\frac{\pi r_{m}}{2 \mu_{m}}\left(\begin{array}{cc}
U_{m n} & V_{m n} \\
Y_{m n} & X_{m n}
\end{array}\right)\left(\begin{array}{l}
A_{m, n} \\
B_{m, n}
\end{array}\right) \\
U_{m n}=\mu_{m} k_{m+1} J_{n}\left(k_{m} r_{m}\right) Y_{n}\left(k_{m+1} r_{m}\right)-\mu_{m-1} k_{m}^{\prime} J_{n}^{\prime}\left(k_{m} r_{m}\right) Y_{n}\left(k_{m+1} r_{m}\right. \\
V_{m n}=\mu_{m+1} k_{m} J_{n}\left(k_{m} r_{m}\right) J_{n}\left(k_{m+1} r_{m}\right)-\mu_{m} k_{m+1} J_{n}\left(k_{m} r_{m}\right) J_{n} \cdot\left(k_{m+1} r_{m}\right. \\
V_{m n}=\mu_{m} k_{m+1} Y_{n}\left(k_{m} r_{m}\right) Y_{n}\left(k_{m+1} r_{m}\right)-\mu_{m+1} k_{m} Y_{n} \cdot\left(k_{m} r_{m}\right) Y_{n}\left(k_{m+1} r_{m}\right.
\end{gathered}
$$


$Y_{m+i n}=\mu_{m+1} k_{m} \gamma_{n}\left(k_{m} r_{m}\right) J_{n}\left(k_{m+1} r_{m}\right)-\mu_{m} k_{m+1} Y_{n}\left(k_{m} r_{m}\right) J_{n} \cdot\left(k_{m+1} r_{m}\right)$

The boundary condition in the external $(\mathrm{m}=\mathrm{M})$ and the first layers, gives

$$
C_{n}=j B_{M, 1, n} \quad A_{M-1, n}-j B_{M-1, n}=a_{n} \quad B_{1 n}=0
$$

The problem can be solved starting with the first layer and $A_{1 n}=1$ and normalizing after all the coeficients.

\section{Inverse problem}

The inverse problem can be solved using a optimization technique combined with the direct method. The process is outlined below.

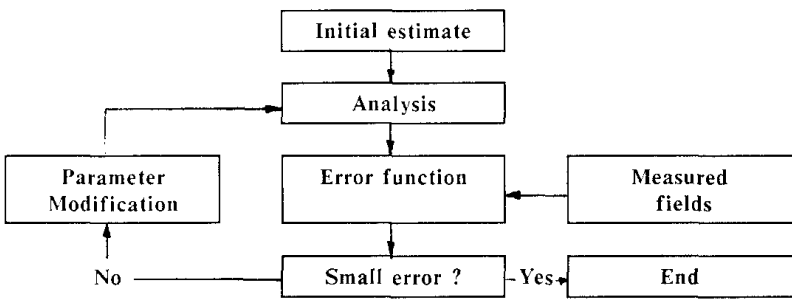

The error function is a cuadratic sum of the difference between the measured fields and the fields calculated with the estimated parameters. The error is multiplied by a weighting factor, taking into acount the validity region for the results

$$
e(\phi, \psi)=\sum h_{1}(\psi)\left|E_{i(\text { mes })}^{s}-E_{i(t h e)}^{s}\right|^{2}
$$

The optimization process is based in the simplex algorithm, that needs multiple evaluation of the error function [4], we reject the methods of gradient because it is necessary to compute the derivative of this function.

\section{Numerical results}

To simulate the reconstruction procedure, we take an object consisting of two concentric cylinders inmmersed in water $(\varepsilon-77.27 \tan \delta=0.1195)$, this body is characterized with the radius, dielectric constant, and loss tangent of the two layers as shown in the first row of table 1 . The measured scattered fields on a 20 $\mathrm{cm}$ diameter semicircular line (fig. 1) are simulated adding gaussian noise to the fields calculated with the direct method at $2.45 \mathrm{GHz}$.

Table 1 shows the reconstruction for several $\mathrm{S} / \mathrm{N}$ ratios in the field measurement; with $\mathrm{S} / \mathrm{N}>20 \mathrm{~dB}$ the accuracy is remarkable and $5 \mathrm{~dB} \mathrm{~S} / \mathrm{N}$ is enough to retrieve the object with an error below $10 \%$ in all parameteres. 
The results have been obtained with a threshold error of $10^{-8}$, requiring around 150 iterations. The computation time is 15 minutes on a Hewlett-Packard series $9000 / 840$ computer.

\begin{tabular}{|c|c|c|c|c|c|c|}
\hline $\mathrm{S} / \mathrm{N}$ & $\mathrm{R}_{1}(\mathrm{~cm})$ & $\epsilon_{1}$ & $\tan \delta_{1}$ & $R_{2}(\mathrm{~cm})$ & $\epsilon_{2}$ & $\tan \delta_{2}$ \\
\hline \hline theory & 3.0000 & 32.00 & 0.200 & 5.000 & 62.580 & 0.050 \\
\hline$\infty$ & 2.9996 & 31.97 & 0.208 & 5.002 & 62.581 & 0.049 \\
\hline 40 & 2.9999 & 32.01 & 0.198 & 5.001 & 62.581 & 0.049 \\
\hline 30 & 3.0038 & 32.07 & 0.194 & 5.005 & 62.662 & 0.051 \\
\hline 20 & 2.9936 & 32.21 & 0.202 & 5.003 & 62.622 & 0.051 \\
\hline 15 & 2.9591 & 32.65 & 0.236 & 5.015 & 62.450 & 0.048 \\
\hline 10 & 2.9955 & 33.58 & 0.211 & 5.012 & 62.080 & 0.040 \\
\hline 5 & 2.8942 & 35.31 & 0.150 & 4.905 & 59.330 & 0.058 \\
\hline
\end{tabular}

Experimental results

A tomographic microwave system [5] operating at $2.45 \mathrm{GHz}$ has been used to measure the scattered field produced by a dielectric cylinder. Fig. 2 shows a diagram of the system, consisting of a circular array of 64 antennas, a coherent microwave transmitter/receiver and control circuitry. Each antenna can be addresed as emitter or receiver using a switching matrix. The cylinder is placed in the center of the geometry and the scattered field produced on an incident cylindrical wave are measured by the half array opposite the emitter element. Fig. 3 shows the comparison between the measured scattered field and the theoretical field correpondig to the optimized cylinder $\epsilon=61.62 \tan \delta-0.38 R=2.23 \mathrm{~cm}$, the reconstruction error is negligible for the radius and below $10 \%$ for the dielectric constant and loss tangent.

References

[1] J. M. Rius, M. Ferrando, et al. Microwave tomography: an algorithm for cylindrical geometries, Electronic Letters, 21 May 1987, pp 564-565

[2] M. Slaney, A. C. Kak, L. E. Larsen, Limitations of imaging with first-order diffraction tomography. IEEE Trans. On Microwave Theory and Techn., August 1984 
[3] Howard E. Bussey, Jack H. Richmond, Scattering by a Lossy Dielectric Circular Cylindrical Multilayer, Numerical Values, IEEE Trans. Antennas and Propagation, Sept 1975, pp 723-725

[4] W.H. Press, B.P. Flannery, S.A. Teukolsky, W.T. Vetterling, The art of scientific Computing, Cambridge University Press, 1986

[5] M. Ferrando, L. Jof re, et al., Cylindrical Microwave Imaging System. IEEE AP-S International Symposium \& URSI Radio Science Meeting, Syracuse, New York, June 6-10, 1988

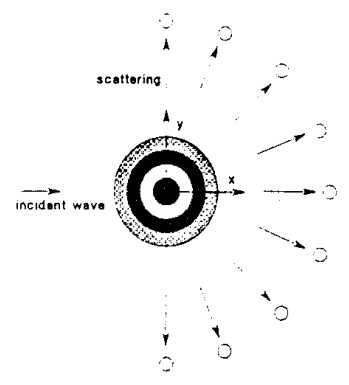

Figure 1.

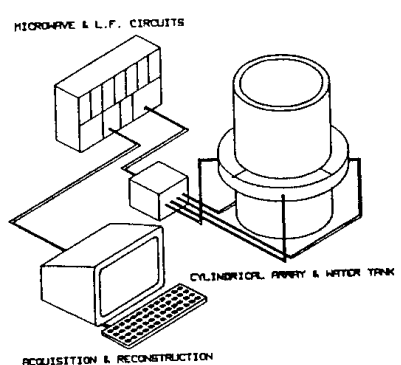

Figure 2. Measurement set-up

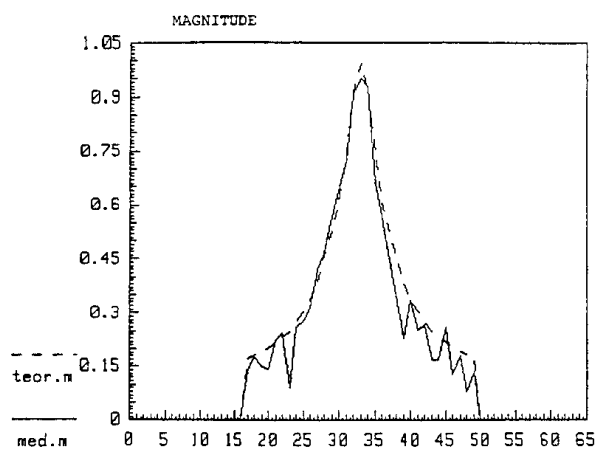

$$
\text { Aritennas }
$$

Figure 3. An experimental gel cylinder optimization. The measured scattered field is shown in solid line. in dotted line the calculated field from the optimized cylinder. 